\title{
meeting point
}

\section{Where do you come from; where do you go? Pluripotency, differentiation and malfunction of stem cells}

\author{
Francesca M. Spagnoli, Daniel Besser \& Salvador Aznar Benitah
}

The international conference 'Stem Cells in Development and Disease' took place in September 2011 at the Max-Delbrück-Center for Molecular Medicine (MDC) in Berlin. It brought together scientists working on different types of stem cell and covered the latest findings in stem cell biology, including the genetic and epigenetic mechanisms of reprogramming, maintenance of pluripotency and differentiation.

\section{$\mathrm{T}$} his outstanding meeting on stem cell biology (http://www.stemcell2011mdc-berlin.de/) was organized by five scientists from the Max-DelbrückCenter for Molecular Medicine (MDC, Germany) and Shin-Ichi Nishikawa (RIKEN, Japan). The excellent line-up of speakers and the 420 international participants combined with the beautiful autumn weather created an inspiring atmosphere for vivid scientific discussions and fruitful interactions. Keynote lectures, invited and selected talks, industry presentations and posters covered a broad range of novel findings in this fast-paced scientific field, covering the state of the art and implications for the therapeutic potential of stem cells; some highlights are covered below.

One of the main challenges in stem cell biology over the past decade has been to unlock the mechanisms that direct the differentiation of embryonic stem cells (ESCs) or induced pluripotent stem cells (iPSCs) into specific cell lineages in vitro. From many possible strategies, the most successful has turned out to be to simply mimic embryonic development. As Janet Rossant (SickKids, Canada) has stated: "If we understand developmental steps in vivo, we can replicate them in a Petri dish" (Rossant, 2011). Thus, scientists have to apply lessons from embryonic development to the guidance of stem cell differentiation in vitro. This requires pinpointing the exact embryonic origin of any cell lineage of interest, and key examples were presented during the meeting.

...apply lessons from embryonic development to the guidance of stem cell differentiation in vitro

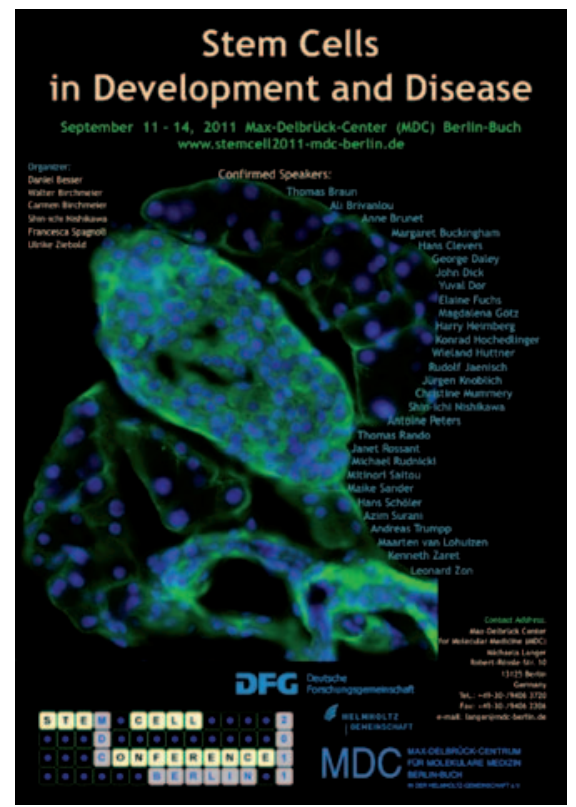

Shin-Ichi Nishikawa addressed the problem by performing an accurate genetic dissection of the network of transcription factors required to make haematopoietic stem cells (HSCs) in the primitive mesoderm of the mouse embryo. Subsequently, he tracked independent progenitor subpopulations and showed their individual contributions to specific haematopoietic and/or endothelial lineages. Leonard Zon (Harvard Medical School, USA) discussed the advantages of using a transparent model organism such as the casper zebrafish to image and model the movement of HSCs in vivo. Transplanting fluorescent cells into this model makes it possible to image single cells and use this as a platform for chemical screens to study the homing and engraftment of HSCs.
Another important example came from Sebastian Arnold (U. Freiburg, Germany). Lineage tracing of eomesodermin-expressing cells in the developing mouse embryo exposed a close relationship between the definitive endoderm and cardiovascular cell lineages, which are separated by differential levels of TGF- $\beta$ signalling. Within the definitive endoderm lineage, Ken Zaret (U. Penn, USA) provided compelling evidence for the existence of two independent embryonic origins of the mammalian liver using embryonic fate mapping. This revealed two progenitor populations-lateral and medialwithin the foregut endoderm that contribute to two different regions-centre and periphery - of the adult liver. Such studies have provided a better understanding of progenitor cell emergence during development, which will aid efforts to control different fates of ESCs or iPSCs.

Janet Rossant's group used transcription factors to restrict human ESCs (hESCs) to definitive-endoderm-specific progenitor populations and then to a liver fate through expression of the hepatic transcription factor HNF4a. Although Rossant has enhanced the existing protocols to differentiate hESCs to liver (or lung), guiding these cells into fully functional and mature cell types in a dish remains a challenge due to marked divergence between the pathways that promote later maturation in human and mouse cells. Maike Sander (U. California San Diego, USA) presented ongoing efforts to differentiate hESCs into functional pancreatic $\beta$-cells. Current protocols now allow the stepwise generation of pancreatic progenitors, but these cells only complete their differentiation and become insulin-producing cells after transplantation into an in vivo environment. 
By using next-generation sequencing approaches such as ChIP-Seq and RNASeq, Sander has characterized the chromatin landscapes of pancreatic cells before and after transplantation to elucidate events that do not take place in vitro and suggest how we might overcome these limitations.

Mitinori Saitou (Kyoto U., Japan) and Azim Surani (U. Cambridge, UK) highlighted exciting advances in the differentiation of germ cells from pluripotent stem cells. Saitou's work demonstrates that the in vitro derivation of primordial germ cell-like cells from mouse ESCs and iPSCs requires the induction of the epiblast-like cell (EpiLC) state in the presence of correct signalling cues. Azim Surani indicated that Prdm14 and one of its targets, the KIf2 transcription factor, provide a potent combination for reprogramming epiblast stem cells to ESCs in response to LIF-Stat3 signalling. Some of the resulting epigenetic changes, such as X-chromosome reactivation and DNA demethylation, resemble those found in early primordial germ cells (PGCs), where Prdm14 and KIf2 expression occurs. However, the response does not completely mimic events in the germ line, where Blimp1/Prdm14 play a combinatorial role in the initiation of germ-line reprogramming. Mehdi Pirouz (MPI for Biophysical Chemistry, Germany) showed that a protein related to the key component of the mitotic spindle assembly checkpoint is required for $\mathrm{G} 2-\mathrm{M}$ cell cycle transition in PGCs; in its absence, PGCs undergo apoptosis.

\section{...a wide variety of intrinsic and extrinsic factors have a major impact on stem cells...}

Several speakers discussed new insights into the impact of epigenetic control on stem cell fate decisions (Sawarkar \& Paro, 2010). By performing in vivo ChIP-Seq on purified hair follicle stem cells and their committed progeny, Elaine Fuchs (Rockefeller U., USA) showed that stemness genes in dormant and active hair follicle stem cells are marked by histone tags associated with active transcription (H3K4me3/ H3K79me3), whereas those required for lineage commitment are silenced by H3K27me3. As stem cells enter the transient amplifying compartment, stemness genes undergo polycomb silencing, while lineage genes become polycomb-derepressed, solidifying fate commitment. By contrast, proliferation genes involved in stem cell self-renewal are not polycomb-regulated, enabling stem cell activation without commitment. Michaela Frye (Wellcome Trust Centre for Stem Cell Research, UK) used ChIP-on-chip to show that Myc binds to the promoters of epidermal differentiation genes to prime them for expression during differentiation. This activity is opposed by the histone deacetylase $\operatorname{Sin} 3$ a through direct deacetylation of $\mathrm{Myc}$, and the epidermal deletion of $\operatorname{Sin} 3$ a results in increased proliferation and differentiation of epidermal stem cells. Conversely, Maarten van Lohuizen (Netherlands Cancer Inst., The Netherlands) demonstrated that deletion of Bmi1 results in hypoproliferation and premature differentiation of mammary gland stem cells. Notably, ectopic overexpression of Bmi1 in the prostate gland, together with ablation of PTEN, results in aggressive prostate carcinomas in mice. Interestingly, these tumours become addicted to Bmi1, opening avenues to pharmacological anti-tumour strategies that inhibit its activity.

Hans Clevers (Hubrecht Inst., The Netherlands) delivered the EMBOsponsored keynote presentation and discussed how intestinal stem cells maintain homeostasis through a stochastic "neutral competition" mechanism. After each daily stem cell division at the intestinal crypt, progeny are randomly pushed either towards differentiation or to remain in the niche as stem cells. Stem cells from a wide variety of epithelial tissues were shown to express high levels of the protein Lgr5, which is activated by the agonist R-Spondin to amplify Wnt signalling. Remarkably, single, isolated Lgr5-positive stem cells can reconstitute a complete, self-sustaining organoid in culture that recapitulates the architecture and functions of the original tissue. This might allow the characterization of pathways that govern stem cell function in several tissues. Konrad Hochedlinger (Massachusetts General Hospital, USA) described how the transcription factor Sox2 also marks stem cells in several murine adult tissues. Intriguingly, Sox2-positive cells do not overlap with the Lgr5 population described by Hans Clevers. Thus Sox2 is not only required for ESC pluripotency, but also for the maintenance of adult stem cells.

John Dick (U. Toronto, Canada) identified another cell surface marker enriched in stem cells, the integrin $a 6$, which allows the isolation of single human HSCs with potent long-term haematopoietic reconstituting activity. Conversely, Leonard Zon showed that prostaglandins cooperate with Wnt to boost HSC engraftment after transplantation. ChIP-Seq analysis of the Wnt transducer TCF4 revealed its localization to chromatin at enhancer regions together with Gata1, Gata2 and Smad1 to define the entire haematopoietic programme. Work from both labs might therefore increase our ability to obtain HSCs and boost their engraftment potential for therapeutic purposes. Furthermore, Leonard Zon indicated that the tissue-specific transcription factors Gata2 in premature cells or Gata1 in mature cells target the BMP-signalling transducer Smad1 to specific promoters, suggesting a general scheme by which tissue-specific transcription factors guide signalling-induced transcription factors to specific target genes.

It is a common understanding that a wide variety of intrinsic and extrinsic factors have a major impact on stem cells and that detailed analyses of these mechanisms are required to control stem cell function in vitro. Extrinsic factors were shown to influence adult stem cell functions. Anne Brunet (Stanford U., USA) described that the transcription factor Foxo3, the activity of which strongly correlates with human longevity, is essential in maintaining the pool of neural stem cells. Salvador Aznar Benitah (Centre for Genomic Regulation, Spain) showed how the circadian molecular clock, which also reads environmental cues, regulates the timely activity of epidermal stem cells. Interestingly, circadian arrhythmia in epidermal stem cells causes premature tissue ageing, and alters the predisposition to develop squamous tumours.

\section{...cell-based therapy is not "around-the-corner", but iPSCs can be used to model diseases and for drug and toxicology screening...}

The generation of cell types that might be useful in the treatment of human diseases is the most challenging goal for stem cell biologists. The 'discovery' of iPSCs (Takahashi \& Yamanaka, 2006), considered a 'magic bullet', should allow the reprogramming of somatic cells; ideally from a patient's own tissues. Our understanding of the mechanisms that underlie the reprogramming of somatic cells has significantly improved 
over recent years. Recent advances were presented in the keynote lecture by Rudolf Jaenisch (MIT, USA) — for example, with respect to the differences between naive and primed pluripotent states and the establishment of naive human pluripotent cells using specific culture conditions. Hans Schöler (MPI for Molecular Biomedicine, Germany) reinforced the notion of culture conditions that stabilize reprogramming and showed the role of chromatin remodelling by the SWI/SNF-like BAF complex during reprogramming.

Many speakers raised the point that cellbased therapy is not "around-the-corner", but iPSCs can be used to model diseases and for drug and toxicology screening in academic and pharmaceutical settings. In fact, pluripotent cells are already proving to be a great tool to screen and test chemical compounds for their effects on differentiation and the toxic effects of substances. Christine Mummery (Leiden U. Medical Center, The Netherlands) has established a robust and reproducible in vitro differentiation protocol for cardiomyocytes. Even though the cells fail to integrate correctly into injured mouse hearts in vivo, they can be used to test drugs known to have side effects on the heart function of patients and to optimize dosages.

The use of iPSCs to understand diseases is another promising area of research, as they can be generated for diseases caused by mutations in genes. Janet Rossant presented exciting data showing the derivation of lung epithelial cells from patients suffering from cystic fibrosis, harbouring mutations in the cystic fibrosis transmembrane conductance regulator (CFTR) gene. Her lab has started to use these cells for drug testing, for example with the CFTR corrector CF-106951 (C18). George Daley (Children's Hospital Boston, USA) derived iPSCs from several disease models, including fragile $\mathrm{X}$ syndrome, Fanconi anaemia and Shwachman Diamond syndrome - a form of ribosomopathy leading to exocrine pancreatic insufficiency. His laboratory has begun to repair the disease genes in vitro and derive relevant cell types for drug testing. Rudolf Jaenisch discussed the use of zinc-finger nucleases and transcription activator-like effector nucleases (TALENs) to repair genetic defects specifically in iPSCs and to obtain isogenic controls that differ only in the disease-relevant gene, excluding unspecific differences due to different genetic backgrounds.

It has been shown that microRNAs (miRNAs) take centre stage in fine-tuning

\section{...microRNAs take centre stage in fine-tuning fate decisions of stem cells...}

fate decisions of stem cells, and they have received considerable interest in recent years for their involvement in development and disease (Bartel, 2009). Important examples were presented in the muscle session. Entry into the myogenic programme in the embryo immediately follows activation of the myogenic determination gene Myf5 in quiescent muscle satellite cells. Margaret Buckingham (Pasteur Institute, France) showed that Myf5 is kept in check through the function of miR-31, which is sequestered with Myf5 mRNA in ribonucleoprotein granules. Tom Rando (Stanford U., USA) showed data implicating miR-489 in the maintenance of satellite cells and identified the DEK oncogene as a relevant target for miR-489. Michael Rudnicki ( $U$. Ottawa, Canada) pointed out that the chromatin remodeller Prdm16 controls the cell fate switch between muscle and brown fat. He established a circuit control in which a Pax7-induced miRNA specifically repressed Prdm16, to regulate a lineage switch from the myogenic to the brown adipogenic lineage.

Other presentations indicated the role of miRNAs beyond myogenesis. Nils Pfaff (Hannover Medical School, Germany) identified a new miRNA family, miR-130a/b, $-301 / \mathrm{b}$ and -721 , that strongly enhances the reprogramming of somatic cells to pluripotent cells. Pfaff showed that their relevant common target is the transcription factor Meox2. Heiko Lickert (Helmholtz Center, Munich, Germany) demonstrated that mesendoderm separation in the mouse embryo is controlled by miR-335. The miRNA is harboured in the mesoderm-specific gene (MEST) and downregulates the endoderm transcription factors Sox 17 and FoxA2.

\section{...we find remarkable conservation of fundamental mechanisms in stem cell biology...}

A clear message from the meeting is that parallel investigations in diverse vertebrate and invertebrate species can accelerate our understanding of stem cell biology towards the goal of treating human disease. Nikolaus Rajewsky (MDC, Germany) presented a remarkable example of the evolutionary conservation of fundamental mechanisms in stem cell biology. Data generated from high-throughput deep sequencing and proteomics of stem cells (neoblasts) of the flatworm Planaria unveiled that many human and mouse pluripotency genes are conserved. Jürgen Knoblich (Institute of Molecular Biotechnology, Austria) provided compelling evidence of the conservation of the mechanisms underlying metric cell division across invertebrate and vertebrate species (Knoblich, 2010). The balance between stem cell self-renewal and differentiation needs to be precisely controlled to ensure tissue homeostasis and prevent tumorigenesis. A genome-wide transgenic RNAi screen in Drosophila neuroblasts allowed Knoblich's group to identify more than 600 genes potentially involved in controlling this balance. His talk focused on the conserved role of Inscuteable in controlling the orientation of asymmetrical cell division in the developing brains of the fly and mouse. Therefore, we find remarkable conservation of fundamental mechanisms in stem cell biology throughout the animal kingdom.

Although the amazing range of topics discussed at the meeting cannot be completely covered here, the participants took away with them a wealth of novel ideas for their ongoing research. The rapid pace of stem cell research promises considerable advances in the field by the time of the next conference. Many thanks to all the participants for having made this meeting a successful event; muchas gracias, es war wunderbar and a presto.

\section{CONFLICT OF INTEREST}

The authors declare that they have no conflict of interest.

\section{REFERENCES}

Bartel DP (2009) Cell 136: 215-233

Knoblich JA (2010) Nat Rev Mol Cell Biol 11: 849-860 Rossant I (2011) Dev Cell 21: 20-23

Sawarkar R, Paro R (2010) Dev Cell 19: 651-661 Takahashi K, Yamanaka S (2006) Cell 126: 663-676

Francesca M. Spagnoli is a junior group leader and Daniel Besser is a team leader at the Max-Delbrück-Center for Molecular Medicine (MDC), Berlin, Germany. Salvador Aznar Benitah is an ICREA researcher and group leader at the Centre for Genomic Regulation (CRG), Barcelona, Spain. E-mails: francesca.spagnoli@mdc-berlin.de; d.besser@mdc-berlin.de; salvador.aznar-benitah@crg.es

EMBO reports (2012) 13, 9-11; published online 9 December 2011; doi:10.1038/embor.2011.241 